\title{
Photosystem II Heterogeneity and Long-Term Acclimation of Light-Harvesting
}

\author{
Pirkko Mäenpää* and Bertil Andersson \\ Department of Biochemistry, Arrhenius Laboratories, University of Stockholm. \\ S-10691 Stockholm, Sweden \\ Z. Naturforsch. 44c, 403-406 (1989); received January 11, 1989 \\ Dedicated to Professor Achim Trebst on the occasion of his 60th birthday \\ Light-Harvesting Chlorophyll a/b-Proteins, Photosystem II, Thylakoid Membrane Organization, \\ Antenna Size Acclimation, Subfractionation \\ The main chlorophyll $a / b$ protein complex of the chloroplast thylakoid membrane is organized \\ into two subpopulations; one inner which is tightly bound to the photosystem II core and one outer \\ which is bound more loosely or peripherally. In this study, changes in the LHC II composition \\ due to long-term light acclimation were analyzed and quantified in spinach thylakoids and isolated \\ stroma lamellae vesicles. The results show that; photosystem II located in the appressed thylakoid \\ regions ( $\alpha$-centres) which have a relatively large antenna size, contains both the inner and outer \\ LHC II with a predominance of the latter $(58-70 \%)$. At low light the antenna size of the $\alpha$-center \\ becomes larger due to a specific increase of the outer pool of LHC II. The antenna size of \\ photosystem II in the stroma thylakoids ( $\beta$-centres) is smaller and contains mainly or only the \\ inner LHC II pool. In contrast to the $\alpha$-centres the $\beta$-centres centres do not undergo adaptive \\ changes in their size in response to long-term changes in the light intensities.
}

\section{Introduction}

A plant is faced with considerable variations in its light environment. These include sudden changes as well as daily and seasonal variations. The ability of a plant to optimize the photosynthetic process and protect the photosynthetic machinery against photodamage is strongly dependent on acclimation mechanisms at the molecular level. These include both short- and long-term acclimations. According to our current knowledge most of the molecular mechanisms for optimizing light-harvesting are targeted to photosystem II and its chlorophyll-proteins [1, 2].

The light-harvesting apparatus of photosystem II in higher plants is extremely complex [3]. There are two chlorophyll $a$ binding proteins of 47 and $43 \mathrm{kDa}$ which are associated with the reaction centre $D_{1} / D_{2}$ protein heterodimer, which in itself only contains a few, probably 4 chlorophyll $a$ molecules [4]. Only some $15-25 \%$ of the chlorophyll is associated with

Abbreviations: CP, chlorophyll-protein; LHC II, main light-harvesting $\mathrm{Chl} a / b$-protein of photosystem II; $\mathrm{CPa}$, chlorophyll $a$-proteins of the photosystem II core.

* Present address: Department of Biology, University of Turku, SF-20500 Turku, Finland.

Reprint requests to B. Andersson.

Verlag der Zeitschrift für Naturforschung, D-7400 Tübingen $0341-0382 / 89 / 0500-0403 \$ 01.30 / 0$ these photosystem II core proteins while the remaining chlorophyll molecules are bound to chlorophyll $a / b$-proteins. Among these the LHC II complex is by far the most abundant carrying up to $75 \%$ of the total chlorophyll of photosystem II. Only minor amounts $(<15 \%)$ are bound to the chlorophyll $a / b$-proteins designated CP29, CP27 and CP24 [3].

Recently, it has become evident that the LHC II is heterogeneous in itself $[5-10]$ and organized into two main subpopulations $[5,6,8]$. There is an inner LHC II pool which is tightly associated with photosystem II. The outer pool is more peripherally bound and undergoes reversible detachment from photosystem II upon protein phosphorylation or at elevated temperatures. This pool of LHC II is therefore involved in the modulation of the photosystem II antenna size under conditions of short-term light stress [2]. In spinach it has been shown that the increase in the antenna size of photosystem II during long-term acclimation to low light conditions is mainly due to an increase in the outer pool of LHC II [11]. Thus, the outer pool of LHC II appears to play a key role for both the short-term and long-term light acclimation of photosystem II [2].

Recently the two pools of LHC II have been isolated to purity by means of preparative isoelectric focusing [12]. The two pools have the same relative chlorophyll content and composition but differ in polypeptide composition. The inner LHC II contains 
only a $27 \mathrm{kDa}$ polypeptide while the outer contains a $27 \mathrm{kDa}$ polypeptide and a $25 \mathrm{kDa}$ polypeptide in a ratio of 1.5:1. The determination of these ratios now allows a quantification of the relative amount of the two subpopulations in spinach leaves exposed to different light regimes.

In this study we have made a quantitative analyse of the long-term light acclimation of the light-harvesting apparatus in relation to both heterogeneity of the LHC II (outer and inner subpopulations) and photosystem II ( $\alpha$ - and $\beta$-centres).

\section{Materials and Methods}

Spinach was grown at $25-30^{\circ} \mathrm{C}$ in a $10 \mathrm{~h} / 14 \mathrm{~h}$ light/ dark cycle at three different light intensities. The photosynthetic photon flux density was $475 \mu \mathrm{mol}$ photons $\mathrm{m}^{-2} \mathrm{~s}^{-1}$ (high light), $300 \mu \mathrm{mol}$ photons $\mathrm{m}^{-2}$ $\mathrm{s}^{-1}$ (medium light) and $185 \mu \mathrm{mol}$ photons $\mathrm{m}^{-2} \mathrm{~s}^{-1}$ (low light). Stacked chloroplast thylakoids were isolated as previously described [13]. Stroma lamellae vesicles were isolated by differential centrifugation following Yeda press fragmentation according to [14] except that $10 \mathrm{~mm} \mathrm{MgCl}_{2}$ was present during the press treatment. The Yeda press homogenate was centrifuged at $40,000 \times g$ for $45 \mathrm{~min}$. The supernatant, containing the stroma thylakoid vesicles, was sedimented at $100,000 \times g$ for $45 \mathrm{~min}$.

Chlorophyll-protein complexes were resolved by mild SDS-PAGE [15]. Slab gels containing an acrylamide gradient of $8-12 \%$ were used. The electrophoresis was run for $1-1.5 \mathrm{~h}$ at $0{ }^{\circ} \mathrm{C}$ applying a constant current of $15 \mathrm{~mA} / \mathrm{gel}$. The relative amount of the chlorophyll-protein complexes was determined by scanning the gels at 650 and $675 \mathrm{~nm}$.
Apo-polypeptides of LHC II were resolved by two-dimensional SDS-PAGE $[5,16]$. In the first dimension the mild SDS-PAGE was applied to resolve the individual chlorophyll-proteins. Bands corresponding to LHC II monomeric and oligomeric forms were excised from the gel and reelectrophoresed under denaturating conditions. The second dimension gels were stained with Coomassie brilliant blue, destained and scanned by a laser densitometer. The relative amount of the apo-polypeptides of LHC II ( $27 \mathrm{kDa}$ and $25 \mathrm{kDa})$ was determined from their absorption peak areas.

\section{Results and Discussion}

By the mild SDS-PAGE the relative amount of chlorophyll associated with photosystem I (CPI), photosystem II $(\mathrm{CPa})$ and the LHC II can be determined in thylakoid material isolated from leaves grown at different light intensities [1, 11]. In this study we have concentrated on analyses of the relative chlorophyll content of LHC II and CPa (Table I). In the unfractionated thylakoids isolated from the low-light spinach there is an increase in the LHC II/ $\mathrm{CPa}$ ratio of 5.5 from the value of 4.0 seen in the high-light spinach. This change is accompanied by a decrease in the chlorophyll $a / b$ ratio. Such an increase in the relative amount of LHC II at low-light intensities is well documented through several previous studies [1].

Table I also gives the $27 / 25 \mathrm{kDa}$ polypeptide ratio for each thylakoid sample. The increased proportion of LHC II in the unfractionated thylakoids is accompanied by a decrease in the $27 / 25 \mathrm{kDa}$ polypeptide ratio. A polypeptide ratio of 3.3 under high light is

Table I. Long-term acclimation in the antenna of photosystem II. Spinach thylakoids were isolated and subfractionated by Yeda press treatment to yield stroma lamellae vesicles. Chlorophyll-proteins were resolved by mild SDS-polyacrylamide electrophoresis. The two apo-polypeptides of LHC II (27 and $25 \mathrm{kDa}$ ) were isolated by two-dimensional electrophoresis and quantified by densitometry. High light 475, medium light 300 and low light $185 \mu$ mol photons $\mathrm{m}^{-2} \mathrm{~s}^{-1}$. LHC II, main chlorophyll $a / b$ protein of photosystem II, CPa, chlorophyll a proteins of photosystem II core.

\begin{tabular}{lcccccc}
\hline & \multicolumn{2}{c}{ High light } & \multicolumn{2}{c}{ Medium light } & \multicolumn{2}{c}{ Low light } \\
& Thylakoids & $\begin{array}{l}\text { Stroma } \\
\text { lamellae }\end{array}$ & Thylakoids & $\begin{array}{l}\text { Stroma } \\
\text { lamellae }\end{array}$ & Thylakoids & $\begin{array}{l}\text { Stroma } \\
\text { lamellae }\end{array}$ \\
& 4.0 & 2.5 & 4.8 & 2.7 & 5.5 & 2.6 \\
LHC II/CPa & 2.9 & 6.0 & 2.8 & 6.1 & 2.6 & 6.1 \\
Chlorophyll $a / b$ & 3.3 & 4.5 & 3.1 & 4.6 & 2.6 & 4.7 \\
27/25 kDa polypeptide & 42 & 55 & 39 & 56 & 30 & 56 \\
Inner LHC II [\%] & 58 & 45 & 61 & 44 & 70 & 44 \\
Outer LHC II [\%] & & & & & & \\
\hline
\end{tabular}


lowered to 2.6 under low light. By using the recently determined polypeptide ratios for the isolated LHC II subpopulations [12] the proportion of two LHC II pools in each thylakoid membrane preparation was calculated. Thus, a polypeptide ratio of 3.3 in the light-grown thylakoids reflects $42 \%$ of inner LHC II and $58 \%$ of outer LHC II. The corresponding calculation from the polypeptide ratios of the medium- and low-light thylakoids gives $61 \%$ and $70 \%$ of the outer LHC II pool, respectively. Since the chlorophyll/protein ratio of the two LHC II subpopulations is the same [12] the calculated percentage values apply both to the protein and chlorophyl masses.

The additional LHC II units incorporated during the lowered light conditions (Table I) can be calculated to have a $27 / 25 \mathrm{kDa}$ polypeptide ratio of $1.52: 1$, if the total amount of $\mathrm{CPa}$ is considered to be fairly constant [11]. Since this value corresponds to the polypeptide ratio of isolated outer LHC II [12] these analyses show that it is only the outer pool that is responsible for the long-term acclimation of the LHC II antenna to low light.

The relative amounts of chlorophyll-proteins and LHC II apo-polypeptides were analyzed in stroma lamellae vesicles isolated from leaves grown at the three different light intensities. The results show that the photosystem II of the stroma lamellae (photosystem $\mathrm{II}_{\beta}[17]$ ) has a different composition and adaptive behaviour compared to bulk photosystem II (predominantly photosystem $\mathrm{II}_{\alpha}$ ) as measured in intact thylakoids (Table I).

(i) The $\mathrm{LHC} \mathrm{II} / \mathrm{CPa}$ ratio is lower, 2.5-2.7 indicative of a smaller chlorophyll $a / b$-antenna.

(ii) The LHC II shows a higher $27 / 25 \mathrm{kDa}$ polypeptide ratio of $4.5-4.7$, indicative of a high proportion of inner LHC II.

(iii) The LHC II antenna of stroma lamellae does not undergo any adaptive changes in response to varying light intensities. Both the $\mathrm{LHC} \mathrm{II} / \mathrm{CPa}$ ratio and the $27 / 25 \mathrm{kDa}$ polypeptide ratio remain constant.

Our observations on isolated stroma lamellae confirm the concept of a smaller antenna size for the photosystem $\mathrm{II}_{\beta}$ centres as compared to the photosystem $\mathrm{II}_{\alpha}$ centres $[1,17,18]$. In addition, we give direct experimental evidence that the antenna of photosystem II in the stroma lamellae have a different composition regarding the LHC II subpopulations due to the high polypeptide ratio of 4.6 (Table I). This ratio

means that $56 \%$ of the LHC II belongs to the inner pool while $44 \%$ belongs to the outer pool. Therefore, despite the small overall LHC II antenna size of photosystem II in the stroma thylakoids (LHC II/ CPa 2.5-2.7, Table I) the size of its inner LHC II pool is about the same as for bulk photosystem II. In other words, the high proportion of inner LHC II compensates for the lower amount of total LHC II. It can therefore be concluded that the small antenna size of photosystem II in the stroma lamellae is due to low amounts of the outer LHC II subpopulation.

At present we can only speculate on the organization of the two subpopulations in the grana and stroma thylakoids (Fig. 1). In the appressed thylakoids the outer LHC II is thought to be physically associated to the photosystem II core and the inner LHC II and provide connectivity between photosystem $\mathrm{II}_{\alpha}$ centres [18]. In contrast, photosystem $\mathrm{II}_{\beta}$ centres are not thought to be connected. It can there-

GRANA

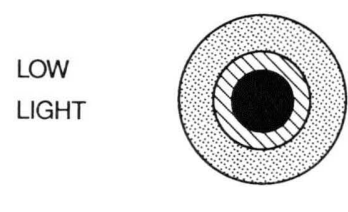

$\mathrm{HIGH}$

LIGHT

IGHT 
fore be argued that the outer LHC II present in stroma thylakoids are not bound to photosystem II [18]. Previous comparisons of the relative antenna size of the $\alpha$ - and $\beta$-forms of photosystem II would favour this notion [18]. Instead these outer LHC II units in the stroma exposed thylakoids may represent LHC II units that are in a phosphorylated state. It has previously been shown that protein phosphorylation leads to migration of outer LHC II units, from the grana thylakoids into the stroma thylakoids [5, 16]. However, these units do not contribute to the functional antenna size of photosystem $\mathrm{II}_{\beta}[19]$ and should not be physically associated with photosystem II. The inner LHC II however is likely to be tightly bound to the core of photosystem $\mathrm{II}_{\beta}$ as in the case of photosystem $\mathrm{II}_{\alpha}$ (Fig. 1). The presence of LHC II in isolated stroma thylakoids do not support a recent freeze-fracture study on a maize mutant suggesting that the bound antenna of photosystem $\mathrm{II}_{\beta}$ does only contain the chlorophyll $a / b$-proteins CP29 and CP24 in addition to CP 47 and CP $43[20,21]$.

Very recent data suggest that photosystem $\mathrm{II}_{\alpha}$ in itself is heterogeneous with respect to antenna size [22]. This would mean that the data in Table I represent the average properties of the LHC II antenna of the $\alpha$-centres. In light of our present data it is likely that the postulated variation within $\alpha$-centres are due to different amounts of outer LHC II.

[1] J. M. Anderson, Annu. Rev. Plant Physiol. 37, 93-136 (1986).

[2] J. M. Anderson and B. Andersson, Trend Biochem. Sci. 13, 351-355 (1988).

[3] G. F. Peter, O. Machold, and J. P. Thornber, in: Plant Membranes: Structure, assembly and function (J. Harwood and T. J. Walton, eds.), pp. 17-31. The Biochemical Society, London 1988.

[4] O. Nanba and K. Satoh, Proc. Natl. Acad. Sci. U.S.A. 84, 109-112 (1987)

[5] U. K. Larsson and B. Andersson, Biochim. Biophys. Acta 809, 396-402 (1985).

[6] R. C. Jennings, K. Islam, and G. Zucchetti, Biochim Biophys. Acta 850, 483-489 (1986).

[7] P. Dunsmuir, Nucleic Acid Res. 13, 2503-2518 (1985).

[8] B. Greene, D. R. Allred, D. Morishige, and L. A. Staehelin, in: Progress in Photosynthesis Research (J. Biggins, ed.), pp. 697-700, Martinus Nijhoff, Dordrecht 1987.

[9] W. Kühlbrandt and J. Barber, Biochim. Biophys. Acta 934, 118-122 (1988).

[10] R. Bassi and D. J. Simpson, Biochim. Biophys. Acta 935, 152-165 (1988).

[11] U. K. Larsson, J. M. Anderson, and B. Andersson, Biochim. Biophys. Acta 894, 69-75 (1987).
Finally, our results provide another new observation in that the LHC II antenna of photosystem II in the stroma thylakoids does not undergo long-term adaptation in response to varying light intensities (Table I). This means that the light adaptation of photosystem II antenna size is restricted to photosystem $\mathrm{II}_{\alpha}$ centres and their peripheral pool of LHC II (Fig. 1). The lack of adaptation of the photosystem $\mathrm{II}_{\beta}$ centres supports the notion that they are not turning over during normal electron transport [18]. Instead some of the photosystem $\mathrm{II}_{\beta}$ centres could represent newly synthesized photosystem II complexes $[18,23]$ which are under assembly. In addition, they may serve as a photoprotected reserve pool of photosystem II [24] able to rapidly replace photosystem II $_{\alpha}$ centres damaged under conditions of strong illumination. In support of that hypothesis we have preliminary observations that the relative amount of photosystem II in stroma lamellae is somewhat higher under high light compared to low light.

\section{Acknowledgements}

This work was supported by the Swedish Natural Science Research Council and the Academy of Finland. We thank Dr. Ulla K. Larsson for fruitful discussions, Ms. Sophie Bingsmark for skilful technical assistance and Ms. Torill Hundal for the illustration work.

[12] M. Spangfort, U. K. Larsson, J. M. Anderson, and B. Andersson, FEBS Lett. 224, 343-347 (1987).

[13] B. Andersson, H.-E. Akerlund, and P.-A. Albertsson, Biochim. Biophys. Acta 423, 122-132 (1976)

[14] B. Andersson and J. M. Anderson, Biochim. Biophys. Acta 593, 427-440 (1980).

[15] J. M. Anderson, J. C. Waldron, and S. W. Thorne, FEBS Lett. 92, 227-233 (1978).

[16] U. K. Larsson, C. Sundby, and B. Andersson, Biochim. Biophys. Acta 894, 59-68 (1987).

[17] J. M. Anderson and A. Melis, Proc. Natl. Acad. Sci. U.S.A. 80, 745-749 (1983).

[18] A. Glazer and A. Melis, Annu. Rev. Plant Physiol. 38, $11-45$ (1987).

[19] U. K. Larsson, E. Ögren, G. Öquist, and B. Andersson, Photobiochem. Photobiophys. 13, 29-39 (1986).

[20] B. A. Greene, D. R. Allred, D. T. Morishige, and L. A. Staehelin, Plant Physiol. 87, 357-364 (1988).

[21] B. A. Greene, L. A. Staehelin, and A. Melis, Plant Physiol. 87, 365-370 (1988)

[22] P.-A. Albertsson and S.-G. Yu, Biochim. Biophys. Acta 936, 215-221 (1988).

[23] A. K. Mattoo and M. Edelman, Proc. Natl. Acad. Sci. 84, 1497-1501 (1987)

[24] P. Mäenpää, B. Andersson, and C. Sundby, FEBS Lett. 215, 31-36 (1987). 